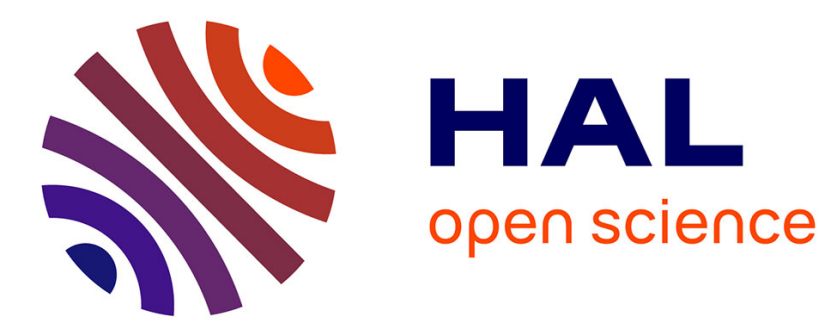

\title{
Influence of temporal pressure constraint on the biomechanical organization of gait initiation made with or without an obstacle to clear
}

\author{
Eric Yiou, Paul Fourcade, Romain Artico, Teddy Caderby
}

\section{- To cite this version:}

Eric Yiou, Paul Fourcade, Romain Artico, Teddy Caderby. Influence of temporal pressure constraint on the biomechanical organization of gait initiation made with or without an obstacle to clear. Experimental Brain Research, 2016, 234 (6), pp.1363-1375. 10.1007/s00221-015-4319-4 . hal-01465685

\section{HAL Id: hal-01465685 \\ https://hal.univ-reunion.fr/hal-01465685}

Submitted on 23 Oct 2017

HAL is a multi-disciplinary open access archive for the deposit and dissemination of scientific research documents, whether they are published or not. The documents may come from teaching and research institutions in France or abroad, or from public or private research centers.
L'archive ouverte pluridisciplinaire HAL, est destinée au dépôt et à la diffusion de documents scientifiques de niveau recherche, publiés ou non, émanant des établissements d'enseignement et de recherche français ou étrangers, des laboratoires publics ou privés. 


\title{
Influence of temporal pressure constraint on the biomechanical organization of gait initiation made with or without an obstacle to clear
}

\author{
Eric Yiou ${ }^{1} \cdot$ Paul Fourcade $^{1} \cdot$ Romain Artico $^{1} \cdot$ Teddy $_{\text {Caderby }}{ }^{2}$
}

\begin{abstract}
Many daily motor tasks have to be performed under a temporal pressure constraint. This study aimed to explore the influence of such constraint on motor perfor-mance and postural stability during gait initiation. Young healthy participants initiated gait at maximal velocity under two conditions of temporal pressure: in the low-pressure condition, gait was self-initiated (self-initiated condition, SI); in the high-pressure condition, it was initi-ated as soon as possible after an acoustic signal (reaction-time condition, RT). Gait was initiated with and without an environmental constraint in the form of an obstacle to be cleared placed in front of participants. Results showed that the duration of postural adjustments preceding swing heel-off ("anticipatory postural adjustments", APAs) was shorter, while their amplitude was larger in RT compared to SI. These larger APAs allowed the participants to reach equivalent postural stability and motor performance in both RT and SI. In addition, the duration of the execution phase of gait initiation increased greatly in the condition with an obstacle to be cleared (OBST) compared to the condi-tion without an obstacle (NO OBST), thereby increasing lateral instability and thus involving larger mediolateral APA. Similar effects of temporal pressure were obtained in NO OBST and OBST.
\end{abstract}

Eric Yiou

eric.yiou@u-psud.fr

1 CIAMS Laboratory, EA 4532, UFR STAPS, University of Paris-Sud, Orsay, France

2 DIMPS Laboratory, EA 4075, UFR STAPS, University of La Réunion, Saint-Denis, France
This study shows the adaptability of the postural system to temporal pressure in healthy young adults initiating gait. The outcome of this study may provide a basis for better understanding the aetiology of balance impairments with the risk of falling in frail popu-lations while performing daily complex tasks involving a whole-body progression.

\section{Introduction}

Gait initiation, the transient period from an upright stance to steady-state walking, is a classical paradigm for studying the coordination between posture, equilibrium and movement. It is composed of a postural phase ending at swing heel-off, during which dynamic phenomena called "anticipatory postural adjustments" (APAs) are developed, followed by step execution phase ending at the time when the swing foot touches the ground (Brenière et al. 1987). APAs associated with gait initiation are typically manifested as a backward and lateral centre-of-pressure shift towards the swing-leg side, which promotes centre-of-mass acceleration in the opposite direction, i.e. forwardly and towards the stance-leg side (Brenière et al. 1987; Brunt et al. 1991; Jian et al. 1993; Elble et al. 1994). APA dynamics along the mediolateral direction are known to be predictive of postural stability reached at the end of gait initiation (Lyon and Day 1997; Mcllroy and Maki 1999; Caderby et al. 2014), while APA dynamics along the anteroposterior direction are predictive of motor performance, in terms of step length and peak centre-of-mass velocity (Brenière et al. 1987; Brenière and Do 1991; Lepers and Brenière 1995). Therefore, any factor affecting the capacity to generate convenient APA may potentially affect both postural stability and motor performance. 
A temporal pressure constraint is known to influence the temporo-spatial features of APA. Specifically, the duration of APA associated with a voluntary arm movement such as shoulder flexion (De Wolf et al. 1998; Nougier et al. 1999) or ball throwing (Ilmane and LaRue 2008) has been shown to be shortened under a condition with high temporal pressure, i.e. when participants have to initiate the task as soon as possible after an imperative "go" signal (referred to as a "reaction-time condition") as compared to a low temporal pressure condition, i.e. when participants initiate the task only when they feel ready (referred to as a "self-initiated condition"). A similar effect of temporal pressure has been reported for tasks involving leg movements such as leg flexion from the erect posture (Yiou et al. 2012b, 2014; Hussein et al. 2013) or gait initiation (Dibble et al. 2004; Delval et al. 2005; Wang et al. 2006). During rapid leg flexion from the erect posture, this shortening of APA duration in the condition with high temporal pressure is compensated by an increase in the anticipatory centre-of-pressure shift towards the swing-leg side so that participants can displace their centre of mass towards the stance-leg side more rapidly (Yiou et al. 2012b, 2014; Hussein et al. 2013), maintaining stability in the final unipodal posture. In addition, performance of the focal movement (maximal leg flexion velocity) remained unchanged as compared to the condition with low temporal pressure. The question of whether the central nervous system is able to develop a similar adaptive strategy during more functional and more complex tasks involving a wholebody progression such as in gait initiation remains to be clarified.

During step initiation as quickly as possible, Wang et al. (2006) reported that the peaks of both the anticipatory mediolateral and backward centre-of-pressure shifts increased when participants performed the task in a reaction-time versus self-initiated condition. However, these authors did not investigate whether these changes in APA amplitude influenced postural stability and/or step performance. Delval et al. (2005) reported a shortening of APA duration when gait initiation was triggered in a reaction-time versus self-initiated condition. For Wang et al. (2006), this shortening was accompanied by an increase in the anticipatory backward centre-of-pressure shift. This increase was, however, not completely sufficient to allow participants to reach equivalent motor performance as in the self-initiated condition. It is noteworthy that the speed instruction provided in this study was to step "rapidly" and not "as quickly as possible" as in previous studies in the literature (Dibble et al. 2004; Wang et al. 2006), which may have masked the true potential of adaptation of APA to temporal pressure. Indeed, the participants may not have developed their maximal effort to reach equivalent motor performance.
In contrast to Wang et al. (2006) and to the studies reported above on leg flexion (Yiou et al. 2012b, 2014; Hussein et al. 2013), Delval et al. (2005) further showed that the decrease in APA duration was not accompanied by an increase in the anticipatory mediolateral centre-of-pressure shift. As stressed by Delval et al. (2005), this discrepancy with the literature might originate from a difference in the way these authors analysed the experimental data. Specifically, these authors did not consider peaks of centre-ofpressure shifts to quantify APA amplitude as is classically done in the literature on gait initiation (e.g. Brenière et al. 1987; Crenna and Frigo 1991; Yiou et al. 2011b; Caderby et al. 2013), rather centre-of-pressure values at the end of APA, i.e. at swing heel-off. Thus, APA amplitude was probably underestimated, making comparison with other studies difficult. In addition, this study did not address the question of whether postural stability was influenced by temporal pressure. While a decline in motor performance may not be dramatic in most daily situations, the maintenance of postural stability, especially along the mediolateral direction, may be critical for body integrity. Indeed, a decline in the control of mediolateral stability is considered a major cause of falling in frail subjects such as the elderly (Maki 1997; Robinovitch et al. 2013), and falls along this direction are seen as a major risk of hip fracture (Smeesters et al. 2001; Kannus et al. 2006).

Hence, the goal of the present study was to investigate the influence of temporal pressure on the biomechanical organization of gait initiation. Gait was initiated in a condition with or without a temporal pressure constraint and with or without an environmental constraint in the form of an obstacle to be cleared placed in front of the participants. The presence of an obstacle to be cleared has been shown to increase the duration of the execution phase of gait initiation (Brunt et al. 1999, 2005; Kim and Brunt 2009) and stepping initiation in reaction to rapid surface translation ("compensatory stepping initiation"; Zettel et al. 2002a, b), thereby potentially increasing mediolateral instability. In the present study, the condition with an obstacle to be cleared was thus used to further challenge the postural balance system, especially in the condition with a high temporal pressure. To date, very few studies focused on the effect of an obstacle to be cleared on the gait initiation process (Brunt et al. 1999, 2005; Kim and Brunt 2009). Brunt et al. (1999) showed that the duration of APA and the peak of anteroposterior ground reaction forces applied to the stance leg (this peak was reached at swing toe-off) were reduced in the obstacle condition compared to the no-obstacle condition. However, these studies could not investigate the relationship between centre-of-pressure and whole-body centre-of-mass as only the ground reaction forces applied to one single limb were recorded during APA. In addition, these studies did not investigate the influence of the 
obstacle to be cleared (or the influence of the temporal pressure) on the control of mediolateral balance and motor performance. Of particular interest, during compensatory stepping initiation, Zettel et al. (2002a, b) showed that the central nervous system anticipates the potential instability associated with obstacle clearance by increasing the amplitude of the mediolateral centre-of-pressure shift so as to maintain balance at swing foot-contact.

Based on this literature review, it can be expected that the temporal pressure will induce an increase in the mediolateral centre-of-pressure shift during APA and that this increase will be exacerbated in the presence of an obstacle to be cleared. Now, because the centre-of-pressure shift is limited by the size of the base of support, it can be questioned whether such a shift is "physically" possible when participants have to clear the obstacle under high temporal pressure. If the amplitude of the mediolateral APA cannot be increased enough to compensate for the shortening of APA duration, a strategy of increasing step width might then be necessary to maintain postural stability (Zettel et al. 2002a, b; Caderby et al. 2014).

The three following hypotheses were tested: (1) the duration of APA associated with gait initiation made with or without an obstacle is shortened under high temporal pressure compared to low temporal pressure. In contrast, the amplitude of APA (in terms of peak centre-of-pressure shift along the anteroposterior and mediolateral direction) is increased to compensate for APA shortening; (2) in the absence of the obstacle, this increase in APA amplitude is sufficient to keep both postural stability and motor performance unchanged compared to low temporal pressure condition; (3) as for the temporal pressure constraint, the presence of the obstacle (considered as an environmental constraint) induces an increase in the mediolateral APA amplitude. It follows that, due to base of support boundaries, a sufficient increase in the mediolateral centre-ofpressure shift during APA may not be "physically" possible when participants have to clear the obstacle under high temporal pressure. If so, a strategy of increasing step width may then be necessary to maintain stability.

\section{Materials and methods}

\section{Participants}

Ten healthy subjects participated in the experiment (eight males and two females, age $23 \pm 4$ years [mean \pm SD], height $175 \pm 8 \mathrm{~cm}$ and weight $70 \pm 11 \mathrm{~kg}$ ). All were free of any known neuromuscular disorder. All participants gave written informed consent after being instructed as to the nature and purpose of the experiment which was approved by the institutional review board. The study conformed to the standards set by the Declaration of Helsinki.

\section{Experimental protocol}

Each participant performed series of gait initiation with their preferred limb in two blocks of conditions: a reactiontime and a self-initiated block. In each block, gait was initiated at maximal velocity, under two conditions of environmental constraint, i.e. in the presence (obstacle condition) or absence (no-obstacle condition) of an obstacle placed in front of the participant. Ten trials were performed in each condition of temporal pressure and environmental constraint $(N=40$ trials). These conditions were randomized across participants to avoid ranking effects. Three minutes of rest was imposed between two successive conditions to avoid fatigue effects. Two familiarization trials were conducted in each condition (not recorded).

In the reaction-time block, the participants initiated gait "as soon as possible" after an acoustic signal was delivered by the experimenter. In the self-initiated block, the participants were instructed to initiate gait only when they felt ready after receiving an "all set" signal; it was made clear to them that the "all set" signal was not a "go" signal and that they could take as much time as they needed to prepare their movements and to perform the movement only when they felt ready. In each condition, the participants initially stood upright with their feet shoulder-width apart, their arms hanging loosely alongside their body and their body weight evenly distributed between their legs. These points were checked visually by the experimenters. The boundaries of each foot in the initial posture were marked on the force plate on which the subjects stood. The experimenters asked the participants to systematically position their feet inside these marks in the initial posture. They directed their gaze ahead towards a target $(4 \mathrm{~cm}$ in diameter) placed at eye level $5 \mathrm{~m}$ in front of the participants. The participants were repeatedly reminded of the task constraint instructions.

In each condition, gait was initiated on a force plate $(600 \times 1200 \mathrm{~mm}$, AMTI, USA) located at the beginning of a 5-m track (Fig. 1). The force plate was embedded in the track and was large enough so that each participant's swing foot systematically landed on it at the end of gait initiation. The participants walked to the end of the track at the instructed velocity and then stood immobile for a few seconds before returning comfortably to their initial posture. In the conditions with an environmental constraint, the obstacle to be cleared was a $20 \mathrm{~cm}$ high $\times 2 \mathrm{~cm}$ deep $\times 60 \mathrm{~cm}$ wide polystyrene block placed at a $20 \%$ body height distance from the participant. The participant's toes served as the reference point for positioning the obstacle. In the noobstacle condition, the participants fixed their gaze towards the target placed in front of them until reaching the end of the track, while in the obstacle condition they were allowed to glance at the obstacle before stepping over it. 
Fig. 1 Schematic illustration of the experimental set-up. Key: (1) walkway; (2) force plate; (3) obstacle; (4) reflective marker; (5) VICON camera; (6) visual target

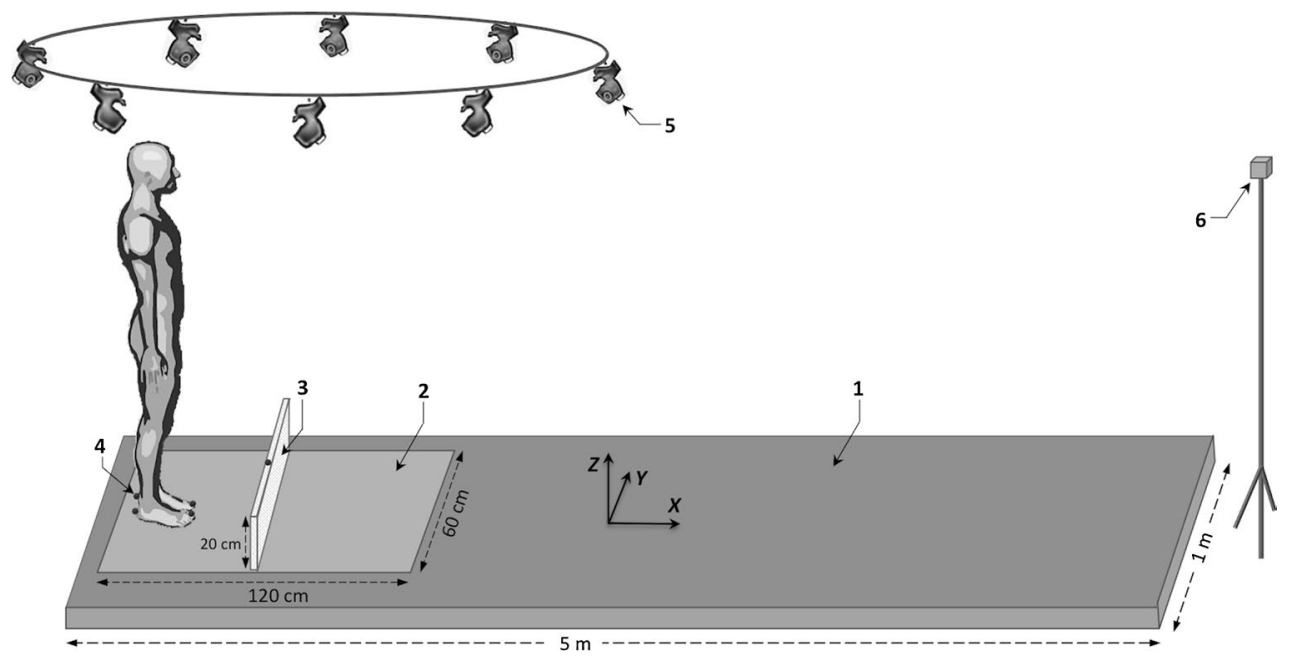

Reflective skin markers ( $9 \mathrm{~mm}$ in diameter) were placed bilaterally at the hallux (toe marker), at the posterior calcaneus (heel marker) and at the middle of the top of the obstacle (obstacle marker; Fig. 1). A V8i VICON eight-camera (Mcam2) motion capture system (Oxford Metrics Ltd, UK) with 64 analogue channels was used to record heel and toe movement and to detect the position of the obstacle. Kinematic and kinetic data were collected simultaneously at a rate of $500 \mathrm{~Hz}$. Data acquisition and stimulus display were controlled by a custom-made program written in Matlab ${ }^{\mathrm{TM}}$ (version 5.3 (R11), The MathWorks Inc., USA).

\section{Data analysis}

Kinematic and force plate data were low-pass-filtered using a Butterworth filter with a $15-\mathrm{Hz}$ (Mickelborough et al. 2000) and a 10-Hz (Caderby et al. 2014) cut-off frequency, respectively. The mediolateral $(\mathrm{yP})$ and anteroposterior (xP) coordinate of the centre of pressure were computed from force plate data as follows:

$$
\begin{gathered}
\mathrm{yP}=\frac{\mathrm{Mx}+\mathrm{Fy} \times d z}{F z} \\
\mathrm{xP}=\frac{-\mathrm{My}+\mathrm{Fx} \times d z}{\mathrm{Fz}}
\end{gathered}
$$

where Mx and My are the moment around the anteroposterior and mediolateral axes, respectively; Fy, Fx and Fz are the mediolateral, anteroposterior and vertical ground reaction forces, respectively; $d z$ is the distance between the surface of the force plate and its origin.

Instantaneous acceleration of the centre of mass along the anteroposterior and mediolateral axes was determined from the ground reaction force according to Newton's second law. Centre-of-mass velocity and displacement were computed by successive numerical integration of centre-of-mass acceleration using integration constants equal to zero, i.e. initial velocity and displacement null (Brenière et al. 1987).

The following instants were determined from biomechanical traces (Fig. 2): gait initiation onset $\left(t_{0}\right)$, swing heel-off, swing foot-off and swing heel-contact. Two $t_{0}$ times were estimated, one for the mediolateral axis and one for the anteroposterior axis. The $t_{0}$ times corresponded to the instants when the $\mathrm{yP}$ or $\mathrm{xP}$ trace deviated 2.5 standard deviations from its baseline value (Caderby et al. 2014). Swing heel-off and toe-off corresponded to the instants when the vertical position of the swing heel marker and the anterior position of the toe marker increased by $3 \mathrm{~mm}$ from their position in the initial static posture. Swing heel-contact corresponded to the instant when the $\mathrm{yP}$ and $\mathrm{xP}$ traces shifted abruptly towards the swing-leg side and forward, respectively.

\section{Dependant variables}

Gait initiation was divided into three phases: APA (from $t_{0}$ to swing heel-off), swing foot-lift (from heel-off to toe-off) and step execution (from swing toe-off to heel-contact). The duration of APA along the mediolateral and anteroposterior axes was computed separately, as the $\mathrm{t}_{0}$ times for these two axes did not necessarily occur simultaneously (Caderby et al. 2014). The reaction time corresponded to the shortest duration between the "go" signal and these two $t_{0}$ instants.

APA amplitude was characterized by the peaks of the backward and lateral centre-of-pressure shift obtained during the APA time window. Centre-of-mass velocity and displacement along the mediolateral and anteroposterior axes were quantified at swing heel-off, toe-off and heel-contact. The mediolateral and anteroposterior centre-of-mass position in the initial upright posture was estimated by averaging the 
Fig. 2 Example of main biomechanical traces obtained for one representative subject initiating gait (one trial) at maximal velocity in the reaction-time condition without obstacle. Anteroposterior direction (panels 1 and 2 from the top). $x^{\prime} M, x P$ centre of mass (CoM) velocity, centre of pressure $(\mathrm{CoP})$ displacement. $x P_{\max }, x^{\prime} M_{H O}, x^{\prime} M_{F O}, x^{\prime} M_{H C}$ peak CoP displacement during APA, CoM velocity at heel-off, at foot-off and at heel-contact. $F$ and $B$ indicate forward and backward. Mediolateral direction (panels 3, 4, 5 from the top). $y^{\prime} M, y M, y P$, $\mathrm{CoM}$ velocity, CoM displacement, $\mathrm{CoP}$ displacement. $y P_{\max }, y^{\prime} M_{H O}$, $y^{\prime} M_{F O}, y^{\prime} M_{H C}, y M_{H C}$ peak of CoP displacement during APA, CoM velocity at heel-off, at foot-off, at heel-contact, and CoM displacement at heel-contact. $S T$ and $S W$ indicate stance limb and swing limb. Vertical dashed lines (top panels) $R T, t_{0}, \mathrm{HO}, \mathrm{FO}, \mathrm{HC}$ GO signal (in the reaction-time condition only), onset variation of biomechanical traces, swing heel-off, swing foot-off and swing heel-contact. Horizontal arrows (bottom of panels): RT, APA, FL, EXE time-windows for reaction time, anticipatory postural adjustments, swing foot-lift, execution phase

centre-of-pressure position during the 250-ms period preceding the "all set" signal (McIlroy and Maki 1999). Gait initiation performance was quantified with the anteroposterior centre-of-mass velocity at heel-contact, step length and step execution duration. Step length corresponded to the distance covered by the heel marker of the swing leg from the initial posture to heel contact. In addition, the vertical distance between (1) the obstacle and the swing heel marker and (2) the obstacle and the swing toe marker was quantified at the time when they passed over the obstacle. For each trial with an obstacle, the shorter of these two vertical distances was reported and corresponded to the "safety distance". The risk of the swing foot striking the obstacle (which might then endanger balance) increased as this safety distance decreased.

As in our previous study on gait initiation (Caderby et al. 2014), an adaptation of the "margin of stability" (MOS) introduced by Hof et al. (2005) was used to quantify mediolateral dynamic stability at heel contact. In the present study, the MOS corresponded to the difference between the mediolateral boundary of the base of support $\left(\mathrm{BOS}_{\mathrm{ymax}}\right)$ and the mediolateral position of the "extrapolated centre of mass" at swing heel-contact $\left(\mathrm{YcoM}_{\mathrm{HC}}\right)$; i.e. MOS = $\mathrm{BOS}_{\mathrm{ymax}}-\mathrm{YcoM}_{\mathrm{HC}}$. Because kinematic data showed that the swing foot-strike was systematically with the heel, BOS $_{\text {ymax }}$ was estimated using the mediolateral position of the heel marker of the swing foot at heel contact. The mediolateral distance between the position of the swing heel marker at heel contact and the position of the stance heel marker at $t_{0}$ represented step width and was representative of the size of the mediolateral base of support.

Based on the study of Hof et al. (2005), the mediolateral position of the extrapolated centre of mass at heel contact $\left(\mathrm{YcoM}_{\mathrm{HC}}\right)$ was calculated as follows:

$\mathrm{YcoM}_{\mathrm{HC}}=\mathrm{yM}_{\mathrm{HC}}+\frac{\mathrm{y}^{\prime} \mathrm{M}_{\mathrm{HC}}}{\omega_{0}}$

\section{Reaction-time without obstacle}

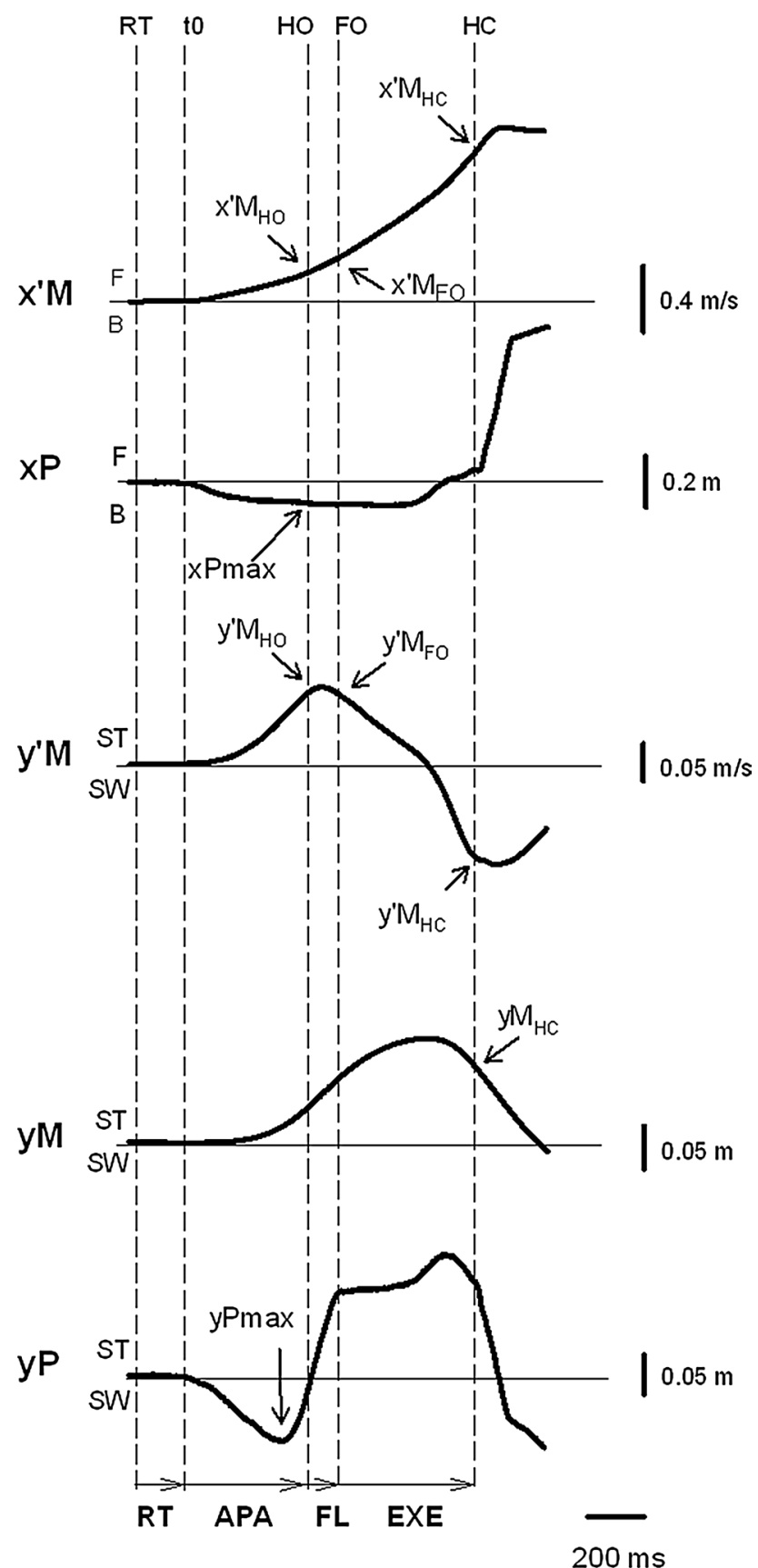

where $\mathrm{yM}_{\mathrm{HC}}$ and $\mathrm{y}^{\prime} \mathrm{M}_{\mathrm{HC}}$ are, respectively, the mediolateral centre-of-mass position and velocity at heel contact, and $\omega_{0}$ is the eigenfrequency of the body modelled as an inverted pendulum calculated as follows:

$\omega_{0}=\sqrt{\frac{g}{l}}$ 
where $g=9.81 \mathrm{~m} / \mathrm{s}^{2}$ is gravitational acceleration and $l$ is the length of the inverted pendulum, which in this study corresponded to $57.5 \%$ of body height (Winter 1990).

Mediolateral dynamic stability at heel contact is preserved on the condition that $\mathrm{YcoM}_{\mathrm{HC}}$ is within $\mathrm{BOS}_{\mathrm{ymax}}$, which corresponds to a positive MOS. A negative MOS indicates mediolateral instability and implies that corrective action (e.g. in the form of an additional lateral step) is required to maintain balance.

\section{Statistics}

Mean values and standard deviations were calculated for each variable in each condition. The normality of data was checked using Kolmogorov-Smirnov test, and the homogeneity of variances was checked using Bartlett's test. An [2 temporal pressure constraint $\times 2$ environmental constraint] ANOVA with repeated measures on each factor was used to test the differences between mean values. The alpha level was set at 0.05 to indicate statistical significance.

\section{Results}

\section{Biomechanical traces}

The time course of the biomechanical traces was globally similar in the different temporal and environmental conditions (Fig. 2). The traces obtained in a single condition are reported in Fig. 2. Swing heel-off was systematically preceded by postural dynamics corresponding to APA. During these APA, the centre-of-pressure displacement reached a peak value backward (cf. the negative variation of the $\mathrm{XP}$ trace in Fig. 2) and towards the swing-leg side (negative variation of the $\mathrm{yP}$ trace), while the centre-of-mass velocity was directed forward (positive variation of the $\mathrm{x}^{\prime} \mathrm{M}$ trace) and towards the stance-leg side (positive variation of the $\mathrm{y}^{\prime} \mathrm{M}$ trace). The mediolateral centre-of-mass velocity trace reached a first peak value towards the stance-leg side at around heel-off. This trace then fell towards the swing-leg side, and a second peak value towards this side was reached a few milliseconds after heel contact. Anteroposterior centre-of-mass velocity increased progressively until it reached a peak value a few milliseconds after heel contact.

\section{Initial posture}

There was no significant effect of the temporal pressure constraint or the environmental constraint on the initial position of the centre of mass along the anteroposterior and mediolateral axes. Also, the distances between the two heel markers and the two toe markers along these two axes were not significantly different across conditions; i.e. foot
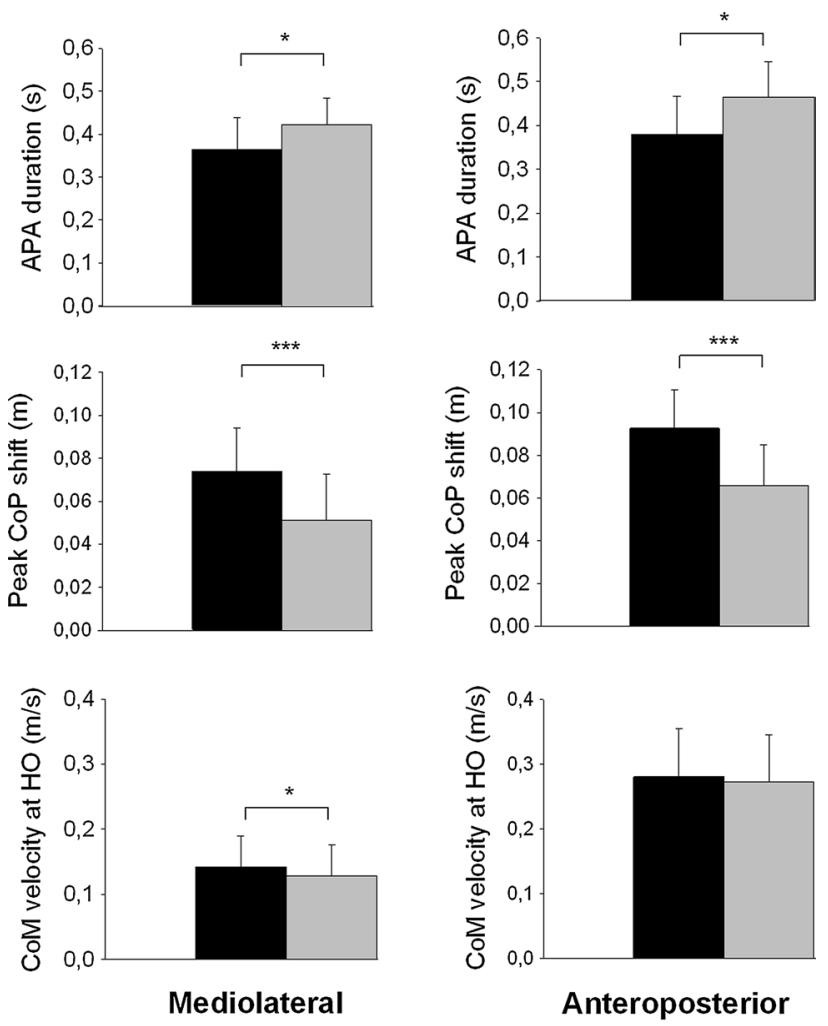

Reaction-time block Self-initiated block

Fig. 3 Main effect of the temporal pressure constraint on selected temporo-spatial parameters of anticipatory postural adjustments (APAs). HO swing heel-off, CoM, CoP centre of mass, centre of pressure. Bars are means \pm one standard deviation (all participants combined). $*, * * *$ Significant main effect of the temporal pressure constraint with $p<0.05$ and $p<0.001$

position and body weight distribution between the legs remained unchanged. The mediolateral and anteroposterior distances between the initial centre-of-pressure position and the swing heel marker were $8.4 \pm 2.0 \mathrm{~cm}$ and $9.3 \pm 2.1 \mathrm{~cm}$, respectively.

\section{Anticipatory postural adjustments}

\section{Temporal pressure constraint}

The temporal pressure constraint had a significant effect on the duration of APA along the mediolateral axis $(F[1,9]=9.15, p<0.05)$. APA duration along this axis was shorter in the reaction-time block than in the selfinitiated block (Fig. 3). In regard to APA amplitude, the results showed a significant effect of the temporal pressure constraint on the peak of anticipatory mediolateral centre-of-pressure shift $(F[1,9]=75.54, p<0.001)$, on the centre-of-mass velocity at swing heel-off $(F[1,9]=6.13$, $p<0.05)$ and at swing toe-off $(F[1,9]=10.72, p<0.01)$. 
The absolute value of each of these variables was greater in the reaction-time block than in the self-initiated block.

The temporal pressure constraint had also a significant effect on the duration of APA along the anteroposterior axis $(F[1,9]=16.19 ; p<0.01)$. APA duration along this axis was shorter in the reaction-time block than in the self-initiated block (Fig. 3). The results also showed a significant effect of the temporal pressure constraint on the peak of anticipatory backward centre-of-pressure shift $(F[1,9]=83.28 ; p<0.001)$; i.e. this peak was greater in the reaction-time block than in the self-initiated block. In contrast, this factor had no significant effect on the anteroposterior centre-of-mass velocity at either swing heel-off or toe-off.

\section{Environmental constraint}

The results showed a significant effect of the environmental constraint on APA duration along the mediolateral axis $(F[1,9]=5.45, p<0.05)$; i.e. APA duration was shorter when gait was initiated in the obstacle block than in the noobstacle block (Fig. 4). There was also a significant effect of the environmental constraint on the peak of the anticipatory mediolateral centre-of-pressure shift $(F[1,9]=49.05$, $p<0.001)$, on the mediolateral centre-of-mass velocity at swing heel-off $(F[1,9]=23.86, p<0.001)$ and at swing toe-off $(F[1,9]=42.00, p<0.001)$. The absolute value of each of these variables was greater in the obstacle block than in the no-obstacle block.

The results further showed a significant effect of the environmental constraint on the peak of backward centreof-pressure shift $(F[1,9]=17.92, p<0.01)$ and on the anteroposterior centre-of-mass velocity at both swing heeloff $(F[1,9]=74.56, p<0.001)$ and toe-off $(F[1,9]=48.26$, $p<0.001$; Fig. 4). In contrast to the mediolateral axis, the absolute value of each of these variables was greater in the no-obstacle block than in the obstacle block. Finally, there was no significant effect of the environmental constraint on the duration of APA along the anteroposterior axis. Also, there was no environmental constraint $\times$ temporal pressure constraint interaction on any of the above-reported variables.

\section{Foot-lift phase}

The results showed a significant effect of the environmental constraint on the duration of the swing foot-lift phase $(F[1,9]=22.28, p<0.001)$. This duration was longer in the no-obstacle block than in the obstacle block. There was no significant effect of the temporal pressure constraint or significant environmental constraint $\times$ temporal pressure constraint interaction on this variable.
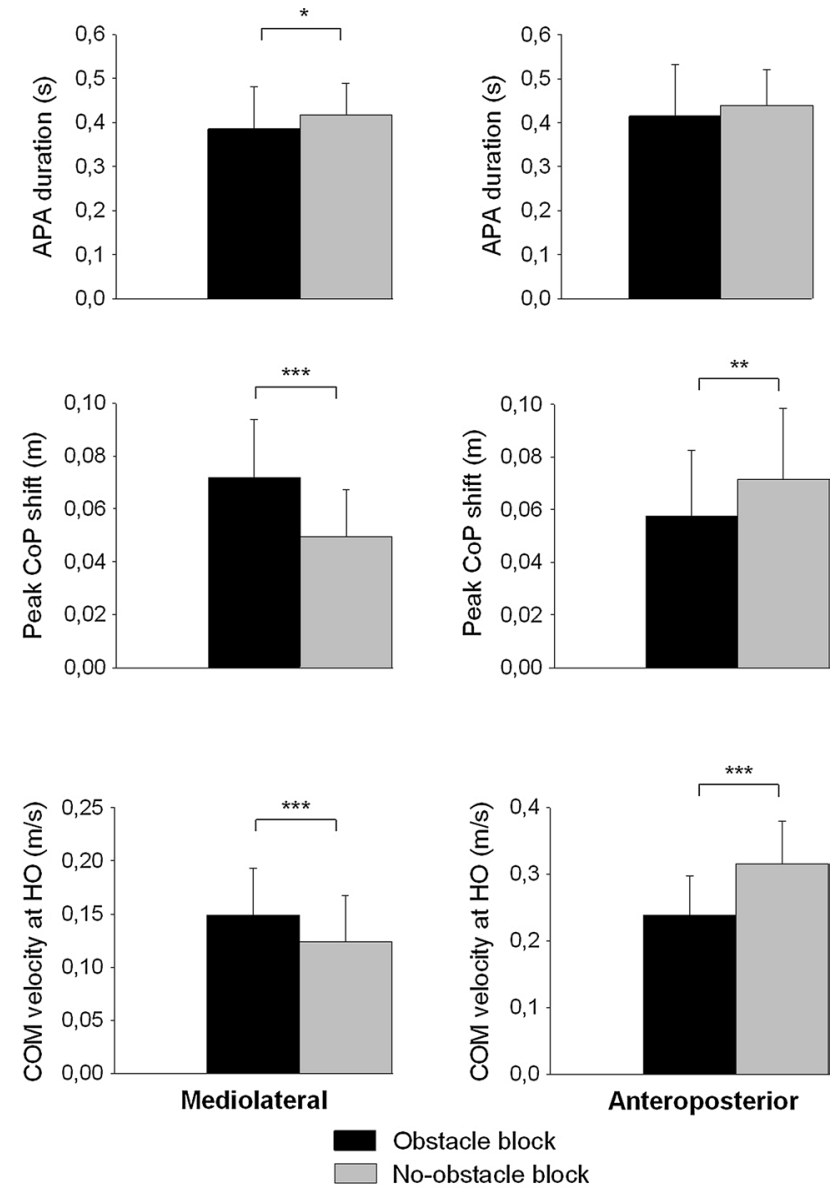

Fig. 4 Main effect of the environmental constraint on selected temporo-spatial parameters of anticipatory postural adjustments (APAs). $\mathrm{HO}$ swing heel-off, $\mathrm{CoM}, \mathrm{CoP}$ centre of mass, centre of pressure. Bars are means \pm one standard deviation (all participants combined). $*, * *, * * *$ Significant main effect of the environmental constraint with $p<0.05, p<0.01$ and $p<0.001$

\section{Postural stability}

The results showed a significant effect of the temporal pressure constraint on mediolateral centre-of-mass velocity $(F[1,9]=8.14, p<0.05)$ and displacement at heel contact $(F[1,9]=7.94, p<0.05$, Fig. 5). Specifically, the mediolateral centre-of-mass velocity was greater in the self-initiated block than in the reaction-time block, whereas centre-of-mass displacement was smaller. In contrast, there was no effect of the temporal pressure constraint on margin of stability (MOS), step width or safety distance (vertical distance between the swing foot and the obstacle). Finally, there was no significant effect of the environmental constraint or significant environmental constraint $\times$ temporal pressure constraint interaction on any of these postural stability-related variables (Fig. 6). 

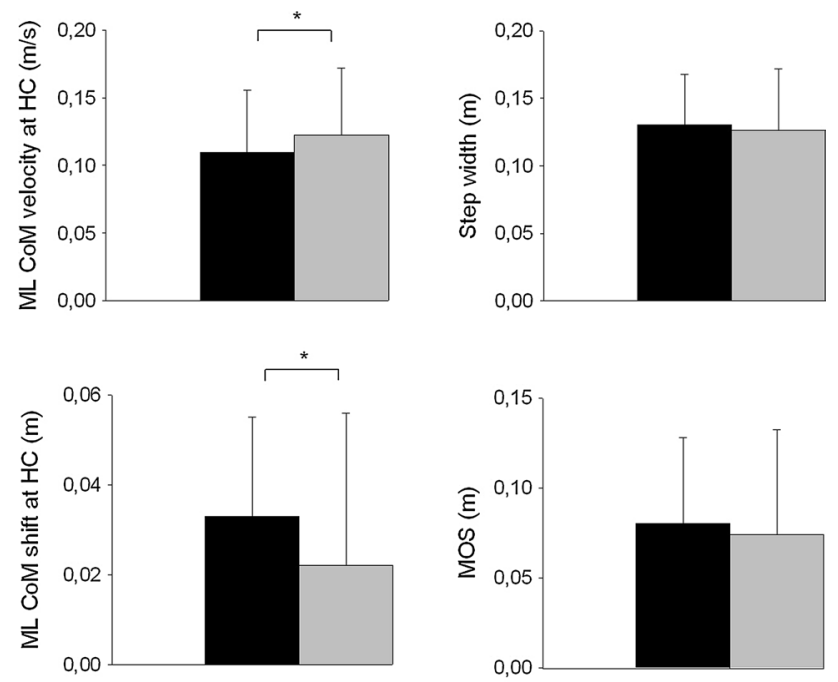

Reaction-time block Self-initiated block

Fig. 5 Main effect of the temporal pressure constraint on selected parameters related to postural stability. $M L$ mediolateral axis. $M O S$ margin of stability (see "Materials and methods" section for details on this parameter). CoM centre of mass. $H C$ swing heel-contact. Bars are means \pm one standard deviation (all participants combined). *Significant main effect of the temporal pressure constraint with $p<0.05$
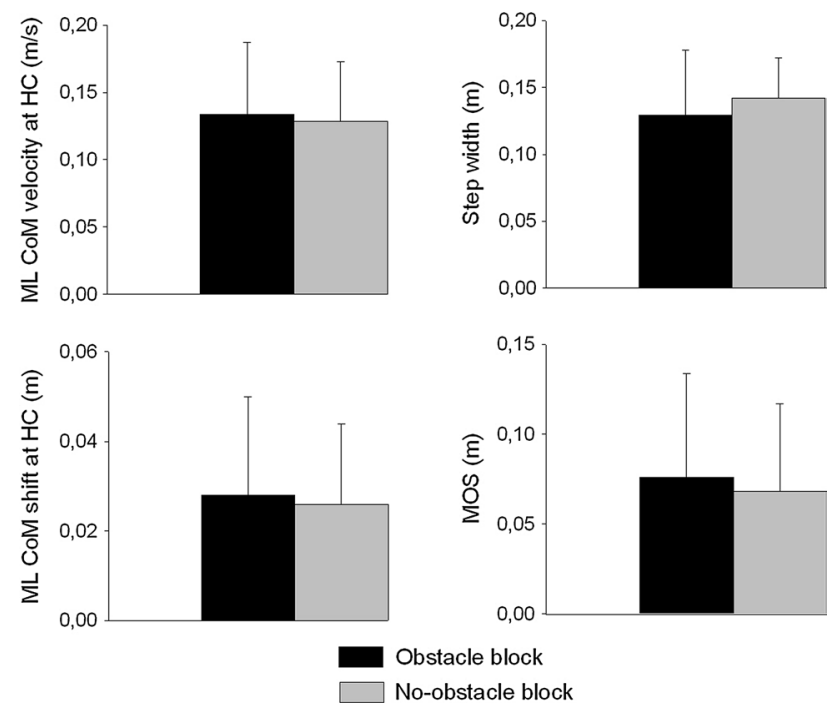

Fig. 6 Main effect of the environmental constraint on selected parameters related to postural stability. $M L$ mediolateral axis. $M O S$ margin of stability (see "Materials and methods" section for details on this parameter). CoM centre of mass. $\mathrm{HC}$ swing heel-contact. Bars are means \pm one standard deviation (all participants combined). Note that there was no significant main effect of the environmental constraint on the reported parameters
Temporal constraint
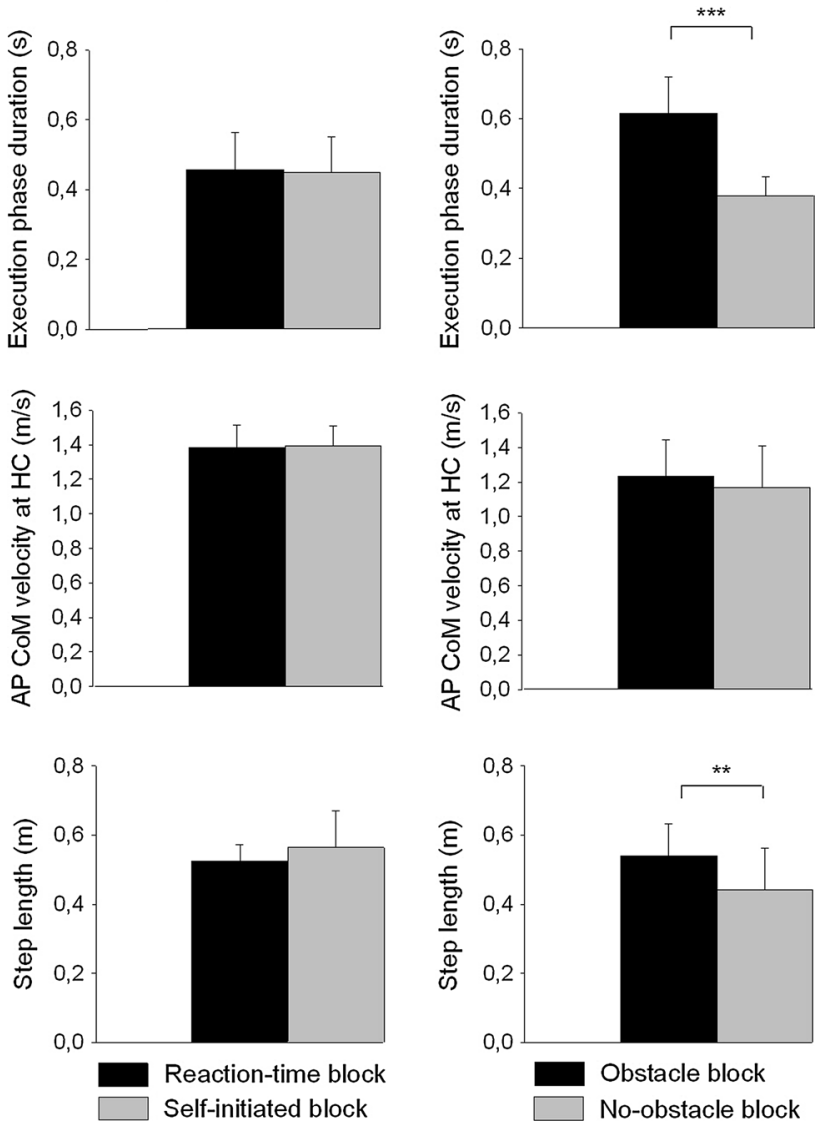

Fig. 7 Main effect of the temporal pressure (left panels) and the environmental (right panels) constraint on selected parameters related to motor performance. Bars are means \pm one standard deviation (all participants combined). CoM centre of mass. AP anteroposterior. $H C$ swing heel-contact. Note that there was no significant main effect of temporal pressure on the reported parameters. $* *, * * *$ Significant main effect of the environmental constraint with $p<0.01$ and $p<0.001$

\section{Motor performance}

There was no significant effect of the temporal pressure constraint on any of the motor performance-related variables (Fig. 7; left panels). In contrast, the results showed a significant effect of the environmental constraint on the duration of the execution phase of gait initiation $(F[1,9]=108.02$, $p<0.001)$ and step length $(F[1,9]=11.64, p<0.01)$. Both variables were greater in the obstacle block compared to the no-obstacle block (Fig. 7; right panels). Of particular note, the duration of the execution phase was almost twice as long in the obstacle block compared to the no-obstacle block. In contrast, this factor had no effect on the anteroposterior centre-of-mass velocity at swing heel-contact. There was no significant environmental constraint $\times$ temporal pressure 
constraint interaction on any of these motor performancerelated variables.

\section{Discussion}

The goal of the present study was to investigate the influence of temporal pressure on the biomechanical organization of gait initiation with or without an obstacle. The addition of an obstacle to be cleared was used to further challenge the postural balance system in the condition with high temporal pressure. The result that, in the condition with high temporal pressure, APA duration was shortened along both the mediolateral and anteroposterior axes is in line with previous studies on gait initiation (Dibble et al. 2004; Delval et al. 2005; Wang et al. 2006), leg flexion (Yiou et al. 2012b; Hussein et al. 2013; Yiou et al. 2014) and arm movement (De Wolf et al. 1998; Nougier et al. 1999; Ilmane and LaRue 2008). As proposed earlier (Yiou et al. 2012b), this shortening probably reflects a strategy to hasten the onset of the voluntary movement-swing heeloff in the present study (Brenière et al. 1987) — in order to meet the temporal constraint of the reaction-time condition to "step as soon as possible following the 'go' signal".

As stressed in the literature, both motor performance (Brenière et al. 1987; Lepers and Brenière 1995) and postural stability (McIlroy and Maki 1999; Yiou et al. 2012a; Caderby et al. 2014) during gait initiation depend on the postural dynamics generated during APA. Hence, because the time available for the organization of APA was shortened by approximately $20 \%$ in the reaction-time block, an alteration of these two important motor features of gait initiation might be expected. However, the results of the present study showed that these motor features remained unchanged across temporal blocks. This finding suggests that, in the block with high temporal pressure, some forms of postural adaptation occurred either during the postural phase preceding APA (i.e. in the initial standing posture) and/or during APA. In the following paragraphs, we discuss the possible adaptive postural strategies implemented by the central nervous system to maintain both motor performance and postural stability unchanged under high temporal pressure constraint.

\section{Influence of temporal pressure on motor performance}

Changing the initial position of the centre of mass is known to influence the development of APA and movement performance (Mille and Mouchnino 1998; Azuma et al. 2007; Dalton et al. 2011). However, the result that, in the present study, there was no main effect of temporal pressure on the position of the centre of mass or on the distance between the feet in the initial posture eliminates this possibility. Therefore, the reported changes in the APA features with temporal pressure cannot be ascribed to any changes in the position of the centre of mass in the initial posture. We thus propose that the increase in the peak of the backward centre-of-pressure shift during APA in the reaction-time block reflects an adaptive response to the reduced APA duration. An anticipatory backward centre-of-pressure shift is known to be responsible for the generation of the initial propulsive forces necessary for whole-body forward progression (Brenière et al. 1987; Lepers and Brenière 1995). By increasing this shift, the participants could generate a greater peak of forward propulsive forces in a shorter time, allowing them to reach an equivalent centre-of-mass velocity at swing heel-off (a similar effect was found at swing foot-off). This velocity, which is representative of the level of forward propulsive forces generated during APA (Brenière et al. 1987; Ito et al. 2003), has been reported to be predictive of both step length and centre-of-mass velocity reached at the end of gait initiation (Brenière et al. 1987; Lepers and Brenière 1995; Michel and Do 2002). Thus, the present results suggest that the participants were able to adapt the amplitude of the anticipatory backward centre-ofpressure shift to the reduced APA duration, so that motor performance could be maintained in terms of step length and progression velocity.

It is interesting to note that a complete adaptation was possible in the reaction-time block because the peak of backward centre-of-pressure shift required for this adaptation was systematically smaller than the distance that it was physically possible to cover during APA. This distance can be estimated using the anteroposterior distance between the position of the heel markers and the position of the centre of pressure in the initial posture (Yiou et al. 2007) and equalled $9.3 \pm 2.1 \mathrm{~cm}$ in the reaction-time block. When gait was initiated without the obstacle to be cleared in the reaction-time condition-i.e. in the condition where the peak of backward centre-of-pressure shift was greatest-the centre of pressure was displaced $9.2 \pm 2.0 \mathrm{~cm}$ from its initial position. This shift therefore almost equalled the distance that it was physically possible to be covered by the centre of pressure during APA (i.e. $9.3 \pm 2.1 \mathrm{~cm}$, as stated above). It follows that a complete adaptation would probably not be possible in the reaction-time condition when APA duration is shortened to a greater extent than that reported, because it would then require a centre-of-pressure shift beyond the base of support, which is obviously not possible. In other words, it appears that the duration of APA in the reactiontime condition was precisely tuned by the central nervous system to reach the minimal possible value allowing complete adaptation, thus revealing a possible functional link between the programming of APA amplitude and APA duration. Interestingly, Jacobs et al. (2009) suggested that the supplementary motor area may contribute to the timing of APA associated with step initiation, whereas APA 
amplitude may be relegated to the primary motor cortex or basal ganglia. The present results illustrate how these neural structures may work together to ensure optimal motor performance and postural stability.

The increase in APA amplitude in the reaction-time block has previously been reported in the literature in young adults (Delval et al. 2005; Wang et al. 2006), in the elderly and in persons with Parkinson's disease performing gait initiation (Dibble et al. 2004). In contrast to the present results, Delval et al. (2005) found that the velocity of the centre of mass at foot contact was lower in the reactiontime block than in the self-initiated block. As stated in the "Introduction" section, the backward centre-of-pressure shift during APA is responsible for the centre-of-mass velocity reached at the end of the gait initiation process (Brenière et al. 1987; Lepers and Brenière 1995). Therefore, the results of Delval et al. (2005) suggest that the greater backward centre-of-pressure shift observed in their study was not sufficient to completely counter the reduction in APA duration. Now, it is noteworthy that, in their study, the decrease in motor performance in terms of anteroposterior centre-of-mass velocity at swing heel-contact was very small $(\sim 9 \%)$ with regard to the drastic reduction in APA duration [ $35 \%$ (as a comparison, it was only $\sim 20 \%$ in the present study)], which leads us to suggest that the adaptive increase in APA amplitude remained very efficient. As argued above, such a drastic reduction in APA duration may not have allowed the development of a complete postural adaptation, probably because it would then imply that the centre-of-pressure shift is displaced beyond the backward boundary of the base of support. Thus, it is possible that the participants in this study "traded" much shorter APA for slightly lower motor performance. In the reaction-time block of the present study, it is not clear why the participants did not use such a trade-off strategy; i.e. why the duration of APA was not shortened as much as in the study by Delval et al. (2005). The finding that the reaction time for initiating gait was even shorter in the present study [112 ms (mean of all reaction-time conditions combined)] than in Delval et al. (2005) (142 ms) rejects the hypothesis that the level of temporal pressure on movement initiation was lower in our study. We propose that the instruction to step "quickly" in the latter study, rather than "at maximal velocity" as in the present study, influenced the strategy the participants used. Indeed, participants in the study of Delval et al. (2005) may not have developed maximal effort to reach equivalent motor performance.

\section{Influence of temporal pressure on postural stability}

In the present study, postural stability was quantified with the MOS (see also Caderby et al. 2014). As for the motor performance, results showed that the temporal pressure did not affect postural stability despite the significant shortening of APA duration along the mediolateral axis. Because it is widely known that postural stability during dynamic motor tasks depends on the capacity of the central nervous system to generate convenient APA (e.g. Bouisset and Do 2008; Yiou et al. 2012a for reviews), this finding suggests that adaptive postural mechanisms occurred during APA along the mediolateral axis to compensate for APA shortening. These mechanisms seem globally similar to those observed along the anteroposterior axis and described above. Specifically, it was found that the decrease in APA duration observed along the mediolateral axis was paralleled by an increase in the peak of the centre-of-pressure shift towards the swing-leg side. This increase was even associated with a greater centre-of-mass velocity at heel-off compared to the self-initiated block, as if the participants "overcompensated" for the reduction in APA duration. This initial centre-of-mass velocity, which was directed towards the stance-leg side, has been shown to minimize centre-ofmass fall velocity towards the swing-leg side at swing footcontact induced by gravity; i.e. it has a stabilizing effect (Lyon and Day 1997; McIlroy and Maki 1999; Caderby et al. 2014). Accordingly, the results of the present study showed that centre-of-mass velocity at foot contact was significantly attenuated in the reaction-time block compared to the self-initiated condition. In contrast, the centre-of-mass shift was greater, reflecting that the lateral fall of the centre of mass was greater in the reaction-time block. Despite these changes in the centre-of-mass position (increase) and velocity (decrease) at swing foot-contact, postural stability estimated with the MOS remained unchanged in the reaction-time and self-initiated blocks. Thus, the present results suggest that the participants were able to adapt the amplitude of the lateral centre-of-pressure shift to the reduced APA duration so that postural stability was maintained. This finding agrees with recent data on gait initiation at different speeds (slow, normal and fast) in a self-initiated condition only (Caderby et al. 2014) which showed that APA duration along the mediolateral axis decreased when the anteroposterior centre-of-mass velocity at swing heelcontact increased. Likely as an attempt at compensation, the peak of the anticipatory lateral centre-of-pressure shift increased, which helped maintain postural stability (also evaluated with the MOS) at swing heel-contact. Collectively, these results are in line with the hypothesis that the MOS is a postural variable that is strongly controlled by the central nervous system (Yiou et al. 2011a, b; Caderby et al. 2014). It is interesting to note that, in the present study, as in the study of Caderby et al. (2014), we observed that the standard deviation values associated with the two kinematical components of the MOS, i.e. the centre-of-mass position and the centre-of-mass velocity at swing foot-contact, were relatively high (cf. Fig. 5). It is possible that these 
relatively large standard deviations, especially observed in the conditions with a high temporal pressure, reflect individual strategies to cope with the mediolateral instability at foot contact, with participants relying more heavily on minimizing one or the other of these two kinematical MOS components in order to maintain the extrapolated centre of mass within the base of support, and thus ensure the condition for dynamic stability.

The adaptation of APA to temporal pressure occurred during gait initiation with and without an obstacle to be cleared. In the condition with an obstacle, postural stability was further challenged by the prolonged duration of the execution phase and the consequent larger fall of the centre of mass towards the swing-leg side (Zettel et al. 2002a, b). To counter this increased risk of postural instability, the participants in the present study developed greater peaks of lateral centre-of-pressure shift during APA. This strategy was efficient since the MOS was not degraded in the obstacle block compared to the no-obstacle block. A similar increase in the spatio-temporal features of lateral APA in the presence of an obstacle to be cleared was previously reported during compensatory stepping forward in reaction to rapid backward force plate translation (Zettel et al. 2002a). However, in this latter study, the greater lateral APA in the obstacle condition as compared to the noobstacle condition was not sufficient to completely counter the increased lateral fall of the centre of mass due to the prolonged duration of the execution phase. Consequently, participants had to use a strategy of increasing step width to recover balance. This type of strategy of step enlargement was not used in the present study. Despite this, the MOS was not degraded in the obstacle block, which shows that the APAs were finely scaled to completely counter the disruptive obstacle effect. This discrepancy with the study of Zettel et al. (2002a) might originate from the difference in the nature of the stepping task (compensatory vs. voluntary in the present study) and/or to a difference in obstacle height ( $15 \%$ of body height vs. $20 \mathrm{~cm}$ in the present study).

The results further showed that there was no temporal pressure $\times$ environmental constraint interaction; i.e. the temporal pressure constraint (and the associated decrease in APA duration) did not induce any additional instability in the obstacle condition. Nor did it alter the participants' ability to clear the obstacle, as revealed by an equivalent safety distance in both conditions. In other words, adding a greater challenge to postural stability with the obstacle did not alter the postural system's ability to adapt APA to temporal pressure. This adaptation to both the presence of an obstacle and the temporal constraint was possible because the width of the base of support in the initial posture was large enough to accommodate the required anticipatory centre-of-pressure shift. Now, it is important to note that the anticipatory lateral centre-of-pressure shift peaked at
$8.7 \mathrm{~cm}$ when gait was initiated in the reaction-time condition with the instruction to clear the obstacle, i.e. in the condition where this peak was greatest. This shift was only $3 \mathrm{~mm}$ greater than the mediolateral distance between the initial position of the centre of pressure and the swing heel marker $(8.4 \mathrm{~cm})$. Therefore, the centre-of-pressure position at the time of the peak value was located between the heel and the lateral boundary of the swing foot (head of the fifth metatarsus); i.e. the peak of centre-of-pressure shift reached almost $100 \%$ of the physically possible mediolateral distance to be covered. Consequently, it is not impossible that, had a higher obstacle been presented $(>20 \mathrm{~cm})$, APA in this condition may have been insufficient to maintain postural stability. A strategy of lateral stepping may then have been required. Future study on this aspect will be undertaken.

\section{Conclusion}

The present study showed that the central nervous system was able to adapt APA amplitude and duration to the temporal pressure constraint so as to hasten swing foot-off and keep both postural stability and motor performance invariant. These results suggest that it might be possible to generalize the postural system's ability to adapt to temporal pressure-which was previously outlined during a leg flexion task (Yiou et al. 2012b; Hussein et al. 2013; Yiou et al. 2014) - to more complex and more common motor tasks of daily living, such as gait initiation made with or without an obstacle to be cleared. This study was carried out in young active adults. It will serve as a basis for future studies in the elderly in the perspective to provide a better understanding of the aetiology of lateral balance impairments with a risk of falling while performing daily tasks involving wholebody progression.

Conflict of interest None of the authors have financial or other conflicts interest in regard to this research.

\section{References}

Azuma T, Ito T, Yamashita N (2007) Effects of changing the initial horizontal location of the center of mass on the anticipatory postural adjustments and task performance associated with step initiation. Gait Posture 26:526-531. doi:10.1016/j. gaitpost.2006.11.203

Bouisset S, Do MC (2008) Posture, dynamic stability, and voluntary movement. Neurophysiol Clin 38:345-362. doi:10.1016/j. neucli.2008.10.001

Brenière Y, Do MC (1991) Control of gait initiation. J Mot Behav 23:235-240. doi:10.1080/00222895.1991.9942034

Brenière Y, Do MC, Bouisset S (1987) Are dynamic phenomena prior to stepping essential to walking. J Mot Behav 19:62-76. doi:10.1 080/00222895.1987.10735400 
Brunt D, Lafferty MJ, McKeon A, Goode B, Mulhausen C, Polk P (1991) Invariant characteristics of gait initiation. Am J Phys Med Rehabil 70:206-212. doi:10.1097/00002060-19910800000009

Brunt D, Liu S-M, Trimble M, Bauer J, Short M (1999) Principles underlying the organization of movement initiation from quiet stance. Gait Posture 10:121-128. doi:10.1016/ S0966-6362(99)00020-X

Brunt D, Santos V, Kim HD, Light K, Levy C (2005) Initiation of movement from quiet stance: comparison of gait and stepping in elderly subjects of different levels of functional ability. Gait Posture 21:297-302. doi:10.1016/j.gaitpost.2004.03.003

Caderby T, Dalleau G, Leroyer P, Bonazzi B, Chane-Teng D, Do MC (2013) Does an additional load modify the anticipatory postural adjustments in gait initiation? Gait Posture 37:144-146. doi:10.1016/j.gaitpost.2012.06.012

Caderby T, Yiou E, Peyrot N, Begon M, Dalleau G (2014) Influence of gait speed on the control of mediolateral dynamic stability during gait initiation. J Biomech 47:417-423. doi:10.1016/j. jbiomech.2013.11.011

Crenna P, Frigo C (1991) A motor programme for the initiation of forward-oriented movements in humans. J Physiol 437:635-653

Dalton E, Bishop M, Tillman MD, Hass CJ (2011) Simple change in initial standing position enhances the initiation of gait. Med Sci Sports Exerc 43:2352-2358. doi:10.1249/ MSS.0b013e318222bc82

De Wolf S, Slijper H, Latash ML (1998) Anticipatory postural adjustments during self-paced and reaction-time movements. Exp Brain Res 121:7-19. doi:10.1007/s002210050431

Delval A, Krystkowiak P, Blatt JL, Labyt E, Destee A, Derambure P, Defebvre L (2005) Caracterisation des ajustements posturaux lors d'une initiation de la marche declenchee par un stimulus sonore et autocommandee chez 20 sujets sains. Neurophysiologie Clinique/Clinical Neurophysiology 35:180. doi:10.1016/j. neucli.2006.01.002

Dibble LE, Nicholson DE, Shultz B, MacWilliams BA, Marcus RL, Moncur C (2004) Sensory cueing effects on maximal speed gait initiation in persons with Parkinson's disease and healthy elders. Gait Posture 19:215-225. doi:10.1016/ S0966-6362(03)00065-1

Elble RJ, Moody C, Leffler K, Sinha R (1994) The initiation of normal walking. Mov Disord 9:139-146. doi:10.1002/mds.870090203

Hof AL, Gazendam MG, Sinke WE (2005) The condition for dynamic stability. J Biomech 38:1-8. doi:10.1016/j.jbiomech.2004.03.025

Hussein T, Yiou E, Larue J (2013) Age-related differences in motor coordination during simultaneous leg flexion and finger extension: influence of temporal pressure. PLoS ONE 8:e83064. doi:10.1371/journal.pone.0083064

Ilmane N, LaRue J (2008) Modulation of anticipatory postural adjustments in a complex task under different temporal constraints. Mot Control 12:330-347

Ito T, Azuma T, Yamashita N (2003) Anticipatory control in the initiation of a single step under biomechanical constraints in humans. Neurosci Lett 352:207-210. doi:10.1016/j.neulet.2003.09.002

Jacobs JV, Lou JS, Kraakevik JA, Horak FB (2009) The supplementary motor area contributes to the timing of the anticipatory postural adjustment during step initiation in participants with and without Parkinson's disease. Neuroscience 164:877-885. doi:10.1016/j.neuroscience.2009.08.002

Jian Y, Winter DA, Ishac MG, Gilchrist L (1993) Trajectory of the body COG and COP during initiation and termination of gait. Gait Posture 1:9-22. doi:10.1016/0966-6362(93)90038-3

Kannus P, Leiponen P, Parkkari J, Palvanen M, Jarvinen M (2006) A sideways fall and hip fracture. Bone 39:383-384. doi:10.1016/j. bone. 2006.01 .148
Kim HD, Brunt D (2009) The effect of a sensory perturbation on step direction or length while crossing an obstacle from quiet stance. Gait Posture 30:1-4. doi:10.1016/j.gaitpost.2009.02.016

Lepers R, Brenière Y (1995) The role of anticipatory postural adjustments and gravity in gait initiation. Exp Brain Res 107:118-124. doi:10.1007/BF00228023

Lyon IN, Day BL (1997) Control of frontal plane body motion in human stepping. Exp Brain Res 115:345-356. doi:10.1007/ PL00005703

Maki BE (1997) Gait changes in older adults: predictors of falls or indicators of fear. J Am Geriatr Soc 45:313-320

McIlroy WE, Maki BE (1999) The control of lateral stability during rapid stepping reactions evoked by antero-posterior perturbation: does anticipatory control play a role? Gait Posture 9:190-198. doi:10.1016/S0966-6362(99)00013-2

Michel V, Do MC (2002) Are stance ankle plantar flexor muscles necessary to generate propulsive force during human gait initiation? Neurosci Lett 325:139-143. doi:10.1016/ S0304-3940(02)00255-0

Mickelborough J, van der Linden ML, Richards J, Ennos AR (2000) Validity and reliability of a kinematic protocol for determining foot contact events. Gait Posture 11:32-37. doi:10.1016/ S0966-6362(99)00050-8

Mille M-L, Mouchnino L (1998) Are human anticipatory postural adjustments affected by a modification of the initial position of the center of gravity? Neurosci Lett 242:61-64. doi:10.1016/ S0304-3940(98)00047-0

Nougier V, Teasdale N, Bard C, Fleury M (1999) Modulation of anticipatory postural adjustments in a reactive and a self-triggered mode in humans. Neurosci Lett 260:109-112. doi:10.1016/ S0304-3940(98)00961-6

Robinovitch SN, Feldman F, Yang Y et al (2013) Video capture of the circumstances of falls in elderly people residing in long-term care: an observational study. Lancet 381:47-54. doi:10.1016/ S0140-6736(12)61263-X

Smeesters C, Hayes WC, McMahon TA (2001) Disturbance type and gait speed affect fall direction and impact location. J Biomech 34:309-317. doi:10.1016/S0021-9290(00)00200-1

Wang Y, Zatsiorsky VM, Latash ML (2006) Muscle synergies involved in preparation to a step made under the self-paced and reaction time instructions. Clin Neurophysiol 117:41-56. doi:10.1016/j.clinph.2005.09.002

Winter DA (1990) Biomechanics and motor control of human movement. Wiley, New York

Yiou E, Hamaoui A, Le Bozec S (2007) Influence of base of support size on arm pointing performance and associated anticipatory postural adjustments. Neurosci Lett 423:29-34. doi:10.1016/j. neulet.2007.06.034

Yiou E, Deroche T, Do MC, Woodman T (2011a) Influence of fear of falling on anticipatory postural control of medio-lateral stability during rapid leg flexion. Eur J Appl Physiol 111:611-620. doi:10.1007/s00421-010-1680-7

Yiou E, Ditcharles S, Le Bozec S (2011b) Biomechanical reorganisation of stepping initiation during acute dorsiflexor fatigue. J Electromyogr Kinesiol 21:727-733. doi:10.1016/j. jelekin.2011.04.008

Yiou E, Caderby T, Hussein T (2012a) Adaptability of anticipatory postural adjustments associated with voluntary movement. World J Orthop 3:75-86. doi:10.5312/wjo.v3.i6.75

Yiou E, Hussein T, Larue J (2012b) Influence of temporal pressure on anticipatory postural control of medio-lateral stability during rapid leg flexion. Gait Posture 35:494-499. doi:10.1016/j. gaitpost.2011.11.015

Yiou E, Hussein T, LaRue J (2014) Influence of ankle loading on the relationship between temporal pressure and motor coordination 
during a whole-body paired task. Exp Brain Res. doi:10.1007/ s00221-014-4003-0

Zettel JL, McIlroy WE, Maki BE (2002a) Can stabilizing features of rapid triggered stepping reactions be modulated to meet environmental constraints? Exp Brain Res 145:297-308. doi:10.1007/ s00221-002-1083-z
Zettel JL, McIlroy WE, Maki BE (2002b) Environmental constraints on foot trajectory reveal the capacity for modulation of anticipatory postural adjustments during rapid triggered stepping reactions. Exp Brain Res 146:38-47. doi:10.1007/ s00221-002-1150-5 